\title{
Electron cloud measurements and simulations for the Brookhaven Relativistic Heavy Ion Collider
}

\author{
W. Fischer, J. M. Brennan, M. Blaskiewicz, and T. Satogata \\ Brookhaven National Laboratory, Upton, New York 11973 \\ (Received 3 September 2002; published 5 December 2002)
}

\begin{abstract}
Intense ion beams in the Brookhaven Relativistic Ion Collider lead to a rise in the vacuum pressure. Electron clouds can contribute to such a process. To measure electron cloud densities the coherent tune shift along the bunch train was observed with different bunch spacings and intensities. From the measured coherent tune shifts, electron cloud densities are computed and compared with densities obtained in electron cloud simulations.
\end{abstract}

DOI: 10.1103/PhysRevSTAB.5.124401

PACS numbers: 29.27.Bd

\section{INTRODUCTION}

The Brookhaven Relativistic Ion Collider (RHIC) consists of two rings, labeled Blue and Yellow. During the RHIC 2001 gold run the number of ions per bunch was continually increased up to the design value of $10^{9}$ at the end of the run. Furthermore, it was attempted to double the number of bunches per ring from 55 to 110. Operation with 110 bunches led to pressure bumps with pressures high enough to prevent operation. In some instances the pressure in the warm sections increased from $10^{-9}$ to $10^{-4}$ Torr $[1,2]$. With the design intensity of $10^{9}$ ions per bunch and 55 bunches in each beam stored at injection, the vacuum system also aborted the beams. Basic machine parameters are listed in Table I; a complete overview can be found in Ref. [3].

Measurements were initiated to characterize the electron cloud buildup and to investigate the possible role of electron clouds in the pressure rise. Since no dedicated electron detectors are currently available in RHIC these measurements were beam based. To obtain an estimate of the electron cloud density, the coherent tune shift along the bunch train was determined. The estimated electron cloud densities can be compared with simulation results.

TABLE I. Machine and beam parameters for gold and protons during RHIC Run 2001/2002, at injection.

\begin{tabular}{lccc}
\hline \hline \multicolumn{1}{c}{ Parameter } & Unit & $\mathrm{Au}^{79+}$ & $p^{+}$ \\
\hline Atomic number $Z$ & $\ldots$ & 79 & 1 \\
Mass number $A$ & $\ldots$ & 197 & 1 \\
Relativistic $\gamma$ & $\ldots$ & 10.5 & 25.9 \\
Harmonic number $h$ & $\ldots$ & 360 & 360 \\
Number of bunches & $\ldots$ & $55 / 110$ & $55 / 110$ \\
Bunch spacing & $\mathrm{ns}$ & $216 / 108$ & $216 / 108$ \\
Ions per bunch $N_{b}$ & $\ldots$ & $10^{9}$ & $10^{11}$ \\
Emittance $\epsilon_{N x, y} 95 \%$ & $\mu \mathrm{m}$ & 10 & 25 \\
Bunch area $S_{95 \%}$ & $\mathrm{eV} \mathrm{s} / \mathrm{u}$ & 0.4 & 1.0 \\
Full bunch length & $\mathrm{ns}$ & 18 & 14 \\
\hline \hline
\end{tabular}

$124401-1$

$1098-4402 / 02 / 5(12) / 124401(8) \$ 20.00$
Such comparisons were also done for the low energy ring of KEKB [4] and the SPS [5,6].

The last RHIC run also allowed the measurement of proton beams. Gold and proton beams have the same number of bunches and approximately the same charge per bunch (see Table I), but their interaction with the rest gas and the wall is different [7]. All tune shift measurements were performed at injection, where gold and proton beams have the same rigidity. The RHIC beam pipe is round almost everywhere. The average beta functions are the same for both planes and so are the beam emittances.

\section{COHERENT TUNE SHIFT MEASUREMENTS}

Coherent tunes shifts along bunch trains at injection were measured with two methods. First, a single beam position monitor (BPM) in each plane recorded the injection oscillations of the last incoming bunch. These BPMs are part of the tune meter system [8]. Typically 1024 turns were recorded and the tunes are obtained from a fast Fourier transform of the coherent beam oscillations. An example is shown in Fig. 1. In this case 110 bunches were injected with an average intensity of $0.3 \times 10^{11}$ protons per bunch. The total tune shift after 110 bunches amounts to $2.5 \times 10^{-3}$. For gold beams and proton beams with large bunch spacing the resolution of these tune measurements was comparable to the tune shifts observed. The tune measurements were improved with a second method.

The orbit system was set to record the injection oscillations of the last incoming bunch in 12 BPMs. In this measurement, all BPM data were filtered and the peak in the spectrum interpolated. In addition, the tune of each bunch could be obtained as an average of the 12 BPM measurements. This procedure is outside the current operational capabilities of the BPM system. A measurement is shown in Fig. 2. In this case a train of 63 bunches was injected with an average intensity $0.65 \times 10^{9}$ gold ions. The vacuum system aborted the fill. Furthermore, a 


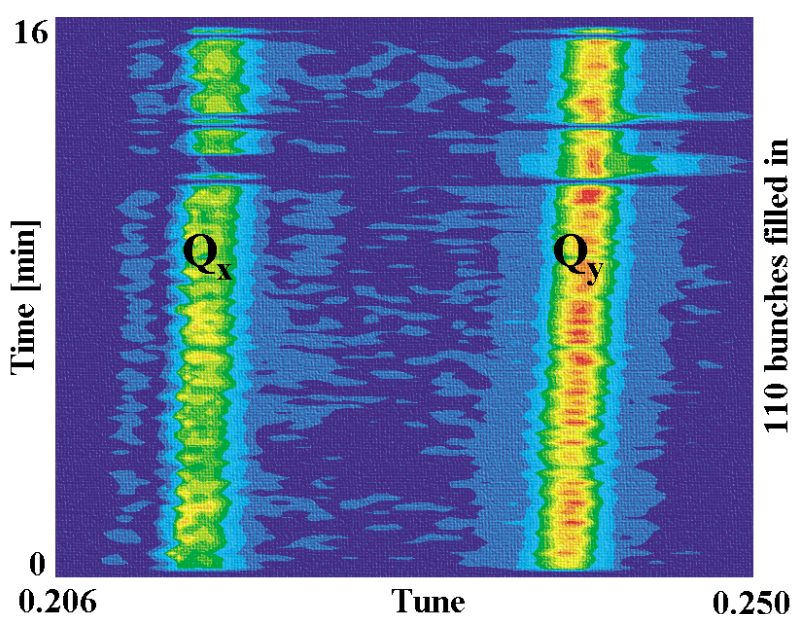

FIG. 1. (Color) Coherent tunes measured along a train of 110 proton bunches with $105 \mathrm{~ns}$ spacing in the Yellow ring. Because of coupling both transverse tunes are visible.

transfer function measurement was tested from which the tunes along the bunch trains can be obtained.

In the measurements, an increase in both transverse tunes was observed, consistent with the existence of an electron cloud. The tune shift is about the same for the horizontal and the vertical plane. In Table II the results of all measurements are summarized. Measured tune shifts are of the order of $10^{-3}$ and are sometimes comparable to the measurement resolution. The data are consistent with the expectation that higher beam intensities and shorter bunch spacing lead to larger tune shifts.
TABLE II. Measured coherent tune shifts $\Delta Q$ along bunch trains. The values given are the difference in tune between bunch 55 and bunch 1 and are averaged over the horizontal and vertical tune shifts. The number of measurements is shown in parentheses.

\begin{tabular}{cccc}
\hline \hline $\begin{array}{c}\text { Bunch spacing } \\
\text { (ns) }\end{array}$ & $\begin{array}{c}\text { Charge per bunch } \\
\left(10^{10} e\right)\end{array}$ & $\begin{array}{c}\text { Tune shift } \Delta Q \\
\mathrm{Au}^{79+} \\
\left(10^{-3}\right)\end{array}$ & $\begin{array}{c}p^{+} \\
\left(10^{-3}\right)\end{array}$ \\
\hline 216 & 7.6 & $1.1(2)$ & $\ldots$ \\
216 & 8.7 & $\ldots$ & $0.3(12)$ \\
108 & 3.0 & $\ldots$ & $1.3(2)$ \\
108 & 5.4 & $1.1(4)$ & $\cdots$ \\
\hline \hline
\end{tabular}

\section{ELECTRON CLOUD DENSITIES}

A bunch passing each turn through a static electron cloud with uniform spatial density $\rho_{e}$ experiences a coherent tune shift [9-11]

$$
\Delta Q_{x, y}=\rho_{e}\left(\frac{r_{p} Z}{\gamma A}\right) \frac{h_{y, x} \beta_{x, y} L}{\left(h_{x}+h_{y}\right)},
$$

where $h_{x, y}$ are the semiaxes of an elliptical chamber, $\beta_{x, y}$ the average beta functions, $L$ the length of the sections with electron clouds, and $r_{p}=1.5347 \times 10^{-18} \mathrm{~m}$ the classical proton radius. In the case of a round beam chamber $\left(h_{x}=h_{y}=h\right)$ and round beams $\left(\beta_{x}=\beta_{y}=\beta\right)$ the tune shifts in both planes are the same $\left(\Delta Q_{x}=\right.$ $\Delta Q_{y}=\Delta Q$ ) and Eq. (1) can be simplified to
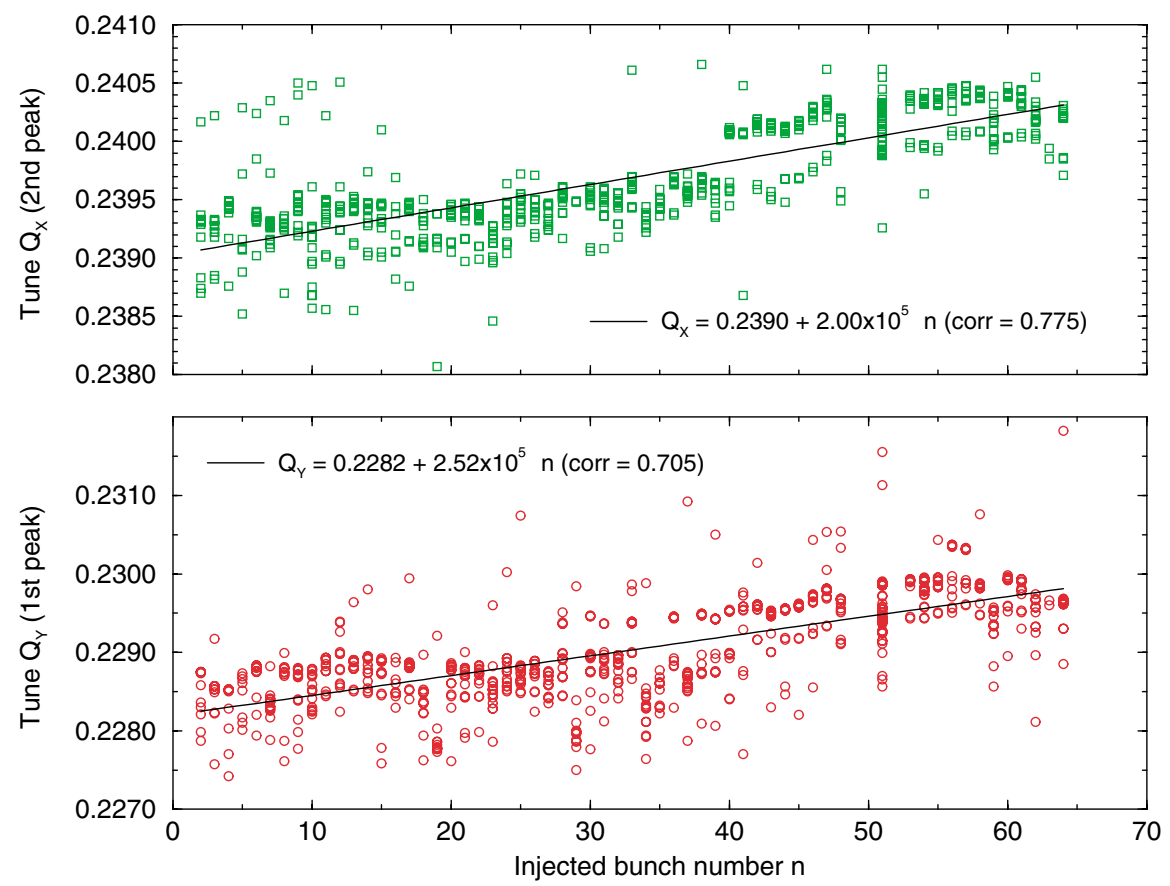

FIG. 2. (Color) Coherent tunes measured along a train of 63 gold bunches with $108 \mathrm{~ns}$ spacing in the Blue ring. Individual dots correspond to the tunes from different BPMs. The solid lines are linear fits to the data. 


$$
\Delta Q=\rho_{e}\left(\frac{r_{p} Z}{\gamma A}\right) \frac{\beta L}{2} .
$$

In the simulation we will see that the time that is needed to reach an electron cloud of constant radial rms size is much shorter than the bunch spacing. We thus assume that the time between bunch passages is also sufficient to create a cylindrically symmetric electron cloud. With a cylindrically symmetric electron cloud and a round beam pipe there are no contributions to the coherent tune shift from the beam surroundings. Assuming that the electron cloud fills the whole beam pipe, the electron line density is $\lambda_{e}=\pi r^{2} \rho_{e}$ where $r$ denotes the average beam pipe inner radius. The charge line density is given by $\lambda_{c e}=$ $\lambda_{e} e$ where $e$ is the electron charge.

We consider the cases of electron clouds in the whole ring and clouds in the warm regions only. The latter is motivated by the fact that significant pressure rises were only observed in a warm region. For relativistic ion beams with the same rigidity the factor $\left(r_{p} Z / \gamma A\right)$ in Eq. (2) is approximately the same. However, gold and proton beams were injected into different lattices, resulting in different values for $\beta$ in both cases.

The relevant machine parameters for all cases and the computed electron cloud densities are shown in Table III. With the assumptions made, one expects charge line densities of 0.2 to $2 \mathrm{nC} / \mathrm{m}$ to account for the measured tune shifts.

Equation (2) gives only a rough estimate for the electron cloud density for two reasons. First, with long bunches the cloud may not be static while the bunch is passing through. In RHIC electrons can perform a few oscillations during a bunch passage. Second, the cloud density may not be distributed uniformly in space. In Ref. [11] the effect of the bunch length on the observed tune shift is investigated analytically and numerically. Significant deviations from Eq. (2) are found for electron

TABLE III. Machine parameters and computed electron cloud densities for different cloud lengths and species.

\begin{tabular}{|c|c|c|c|}
\hline Parameter & Unit & $\mathrm{Au}^{79+}$ & $p^{+}$ \\
\hline Tune shift $\Delta Q$ & $10^{-3}$ & 1.1 & 1.3 \\
\hline$r$ whole ring & $\mathrm{m}$ & \multicolumn{2}{|c|}{0.04} \\
\hline$r$ warm regions only & $\mathrm{m}$ & \multicolumn{2}{|c|}{0.06} \\
\hline$\beta$ whole ring & $\mathrm{m}$ & 30 & 36 \\
\hline$\beta$ warm regions only & $\mathrm{m}$ & 42 & 76 \\
\hline$L$ whole ring & $\mathrm{m}$ & \multicolumn{2}{|c|}{3834} \\
\hline$L$ warm regions only & $\mathrm{m}$ & \multicolumn{2}{|c|}{700} \\
\hline$\rho_{e}$ whole ring & $10^{11} \mathrm{~m}^{-3}$ & 3.3 & 2.9 \\
\hline$\rho_{e}$ warm regions only & $10^{11} \mathrm{~m}^{-3}$ & 12.8 & 7.6 \\
\hline$\lambda_{e}$ whole ring & $10^{9} \mathrm{~m}^{-1}$ & 1.6 & 1.4 \\
\hline$\lambda_{e}$ warm regions only & $10^{9} \mathrm{~m}^{-1}$ & 14.5 & 8.6 \\
\hline$\lambda_{c e}$ whole ring & $\mathrm{nC} \mathrm{m}^{-1}$ & 0.26 & 0.22 \\
\hline$\lambda_{c e}$ warm regions only & $\mathrm{nC} \mathrm{m}^{-1}$ & 2.32 & 1.38 \\
\hline
\end{tabular}

clouds of a size comparable to the beam size while the equation holds for electron clouds large compared to the beam size. In the simulations reported in Sec. V it is found that the electron cloud is much larger than the beam size. This was also found in a RHIC simulation with another code [12]. A transversely large electron cloud, filling most of the beam pipe, is also a good approximation for a cloud with uniform spatial density. Thus Eq. (2) should give a useful estimate for the electron cloud densities.

\section{ELECTRON CLOUD SIMULATIONS}

The computer code NCSEC used here, and its predecessor CSEC, was written by one of the authors (M. B.) to study both the effects of electron gap survival and the electric fields generated by the electrons [13]. They were used previously for the PSR [14] and the SNS [15].

The code assumes an electron cloud that is cylindrically symmetric within a round, straight vacuum chamber, without an external magnetic field. The positively charged beam is also assumed to be round but it can have an offset to the beam pipe center (see Fig. 3). Longitudinal electric fields are ignored, since they produce velocities small compared to the beam velocity. The spatial distribution of the electron cloud is modeled as a sum of $N_{\text {macro }}$ cylindrical shells which serve as macroparticles. This is also shown in Fig. 3. The macroparticle shells can have an angular momentum.

The evolution of the cloud is computed by accelerating the shells and creating secondary electrons when the macroparticles hit the wall. In addition, electrons are created either at the wall or in the beam pipe with a generation rate proportional to the instantaneous beam

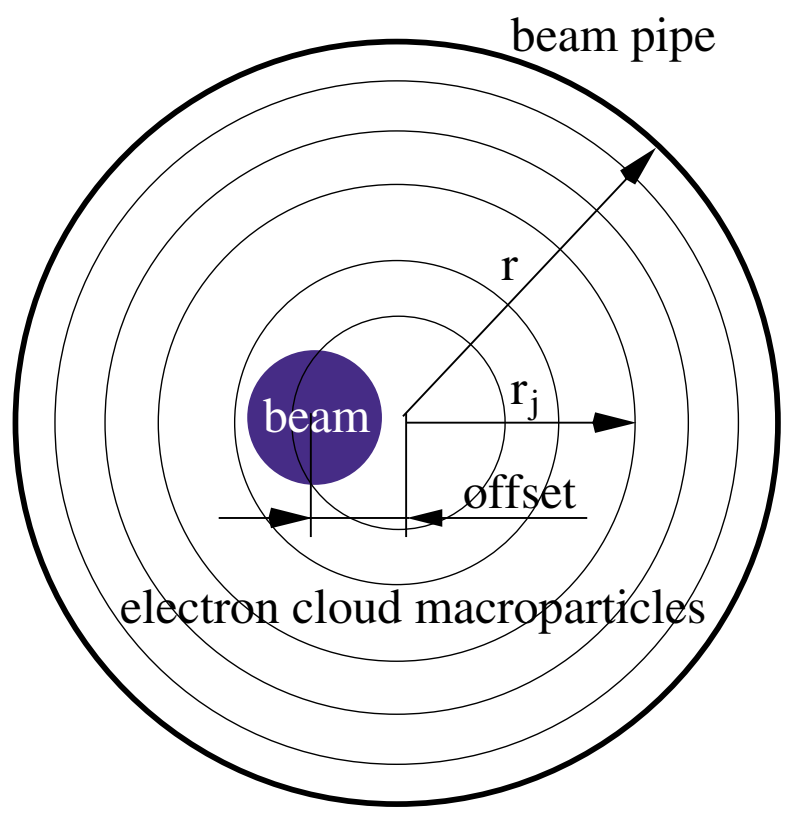

FIG. 3. (Color) Geometry used in the electron cloud simulations. 
line density. The generation rate must be estimated outside the program from processes such as rest gas ionization or beam loss-driven electron generation.

The acceleration of shell $j$, with radius $r_{j}$, due to shell $k$, with radius $r_{k}$ is taken to be nonzero only if $r_{j}>r_{k}$, since no electric field exists inside a uniformly charged cylinder. In this case the acceleration is

$$
\ddot{r}_{j}=2 r_{e} c^{2} \lambda_{k} \frac{r_{j}}{d^{2}+r_{j}^{2}},
$$

where $r_{e}$ is the classical electron radius, $c$ is the speed of light, and $\lambda_{k}$ is the electron line density of shell $k$. The smoothing length $d$ removes singularities for $r_{j} \approx 0$. It is typically an order of magnitude smaller than the beam size. The electric field due to the ion beam has the same form as that due to electrons at $r=0$, with $\lambda_{e}$ being replaced by the instantaneous beam line density multiplied by the ion charge state, $Z \lambda_{b}$.

The time dependence of the instantaneous beam line density is given by

$$
\lambda_{b}(t)=\lambda_{b, \text { peak }}\left(1-\frac{t^{2}}{\tau^{2}}\right)^{n},
$$

where $n$ can be chosen to fit the measured longitudinal beam profile. For large $n$ Eq. (4) approximates a Gaussian beam profile. $\tau$ is a measure for the beam length. The longitudinal bunch profile of a single bunch or a bunch train with gap can also be read in from an external file.

The beam is typically divided into several thousand longitudinal slices $N_{\text {slice }}$, and the electron cloud is updated with every longitudinal slice. Electron macroparticles can carry different charges with a minimum and a maximum charge defined. Macroparticle numbers range from dozens to hundreds of thousands.

The generation of secondary electrons follows largely a model that is presented in Ref. [16]. When an electron macroparticle with energy $E$ hits the wall, it is first determined whether the electron is reflected or generates secondary electrons. In the following, $x_{r}$ denotes a random number out of a uniform distribution between zero and 1 . The electron is reflected if

$$
x_{r 1}<P_{\infty}+\left(P_{0}-P_{\infty}\right) e^{-E / E_{\text {reflect }},}
$$

where $P_{0}, P_{\infty}$, and $E_{\text {reflect }}$ are input parameters that should be determined in measurements [16-18]. $P_{0}$ and $P_{\infty}$ are the probabilities of reflection at zero and large energy, respectively.

If the electron macroparticle is reflected, it can be reflected elastically or it can be rediffused. It is rediffused if

$$
x_{r 2}<P_{\text {rediffuse }},
$$

where $P_{\text {rediffuse }}$ is an input parameter. Otherwise it is elastically reflected. In the former case the energy of the outgoing electron macroparticle is $x_{r 3} E$; in the latter case it is $E$.

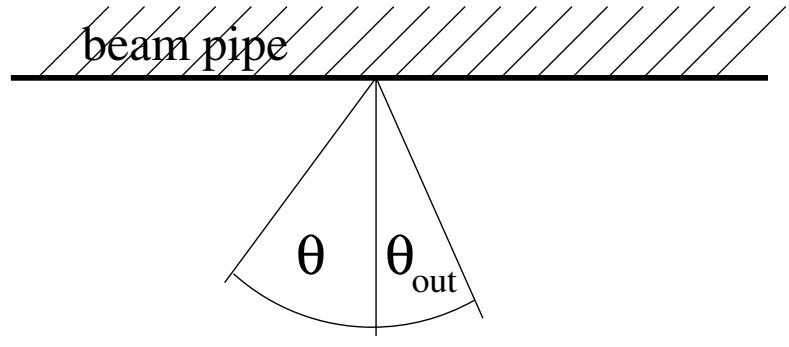

FIG. 4. Definition of angle $\theta$.

If the electron macroparticle is not reflected, it generates secondary electron macroparticles with the emission yield $\delta$ given by

$$
\delta(E)=\delta_{\max } \times 1.1\left(\frac{1-\exp \left[-2.3\left(E / E_{\max }\right)^{1.35}\right]}{\left(E / E_{\max }\right)^{0.35}}\right) .
$$

$\delta_{\max }$ and $E_{\max }$ are input parameters. The line density of the generated macroparticle is

$$
\lambda_{k, \text { out }}=\lambda_{k} \delta(E) e^{\alpha_{\delta}(1-\cos \theta)},
$$

where $\alpha_{\delta}$ is an input parameter and $\theta$ is the incident angle

\begin{tabular}{|c|c|c|c|}
\hline Parameter & Unit & $\mathrm{Au}^{79+}$ & $p^{+}$ \\
\hline Bunch spacing & ns & \multicolumn{2}{|c|}{108} \\
\hline Beam offset & $\mathrm{mm}$ & \multicolumn{2}{|c|}{0} \\
\hline Bunches & $\cdots$ & \multicolumn{2}{|c|}{55} \\
\hline rms beam radius & $\mathrm{mm}$ & 2.2 & 2.4 \\
\hline Pipe radius & $\mathrm{mm}$ & 60 & 40 \\
\hline Electrons generated/bunch & $\cdots$ & 4000 & 100 \\
\hline Electron generation radius & $\mathrm{mm}$ & 60 & 2.4 \\
\hline Full bunch length & ns & 18 & 14 \\
\hline Bunch shape parameter $n$ & $\cdots$ & 3 & 3 \\
\hline Bunch charge & $\mathrm{nC}$ & 13 & 16 \\
\hline Longitudinal slices & $\cdots$ & \multicolumn{2}{|c|}{5000} \\
\hline Macroparticles, initially & $\cdots$ & 250 & 25 \\
\hline Smoothing length $d$ & $\mathrm{~mm}$ & \multicolumn{2}{|c|}{0.1} \\
\hline$\lambda_{c e}$, initial & $\mathrm{pC} \mathrm{m}^{-1}$ & \multicolumn{2}{|c|}{1.6} \\
\hline$P_{0}[16,17]$ & $\cdots$ & \multicolumn{2}{|c|}{0.6} \\
\hline$P_{\infty}$ & $\cdots$ & \multicolumn{2}{|c|}{0.2} \\
\hline$E_{\text {reflect }}$ & $\mathrm{eV}$ & \multicolumn{2}{|c|}{60} \\
\hline$P_{\text {rediffuse }}$ & $\cdots$ & \multicolumn{2}{|c|}{0.5} \\
\hline$\delta_{\max }$ & $\cdots$ & \multicolumn{2}{|c|}{2.3} \\
\hline$E_{\max }$ & $\mathrm{eV}$ & \multicolumn{2}{|c|}{300} \\
\hline$E_{\text {secondary }}$ & $\mathrm{eV}$ & \multicolumn{2}{|c|}{20} \\
\hline$\alpha_{\delta}$ & $\cdots$ & \multicolumn{2}{|c|}{0.5} \\
\hline$\alpha_{\theta}$ & $\cdots$ & \multicolumn{2}{|c|}{1.0} \\
\hline
\end{tabular}
relative to the surface normal (see Fig. 4). The incident

TABLE IV. List of input parameters for electron cloud simulations. For gold and proton beams reference cases are presented with design intensity and twice the design bunch number. 
angle $\theta$ is determined by the macroparticle shell's radial velocity and angular momentum. If the line density is below the set limit, the macroparticle is dropped. If the line density is above the set limit, more than one macroparticle is generated. The energy of the generated macroparticles is

$$
E_{\text {out }}=E_{\text {secondary }} \sqrt{\tan \left(\frac{\pi}{2} x_{r 4}\right)} .
$$

$E_{\text {secondary }}$ is an input parameter.
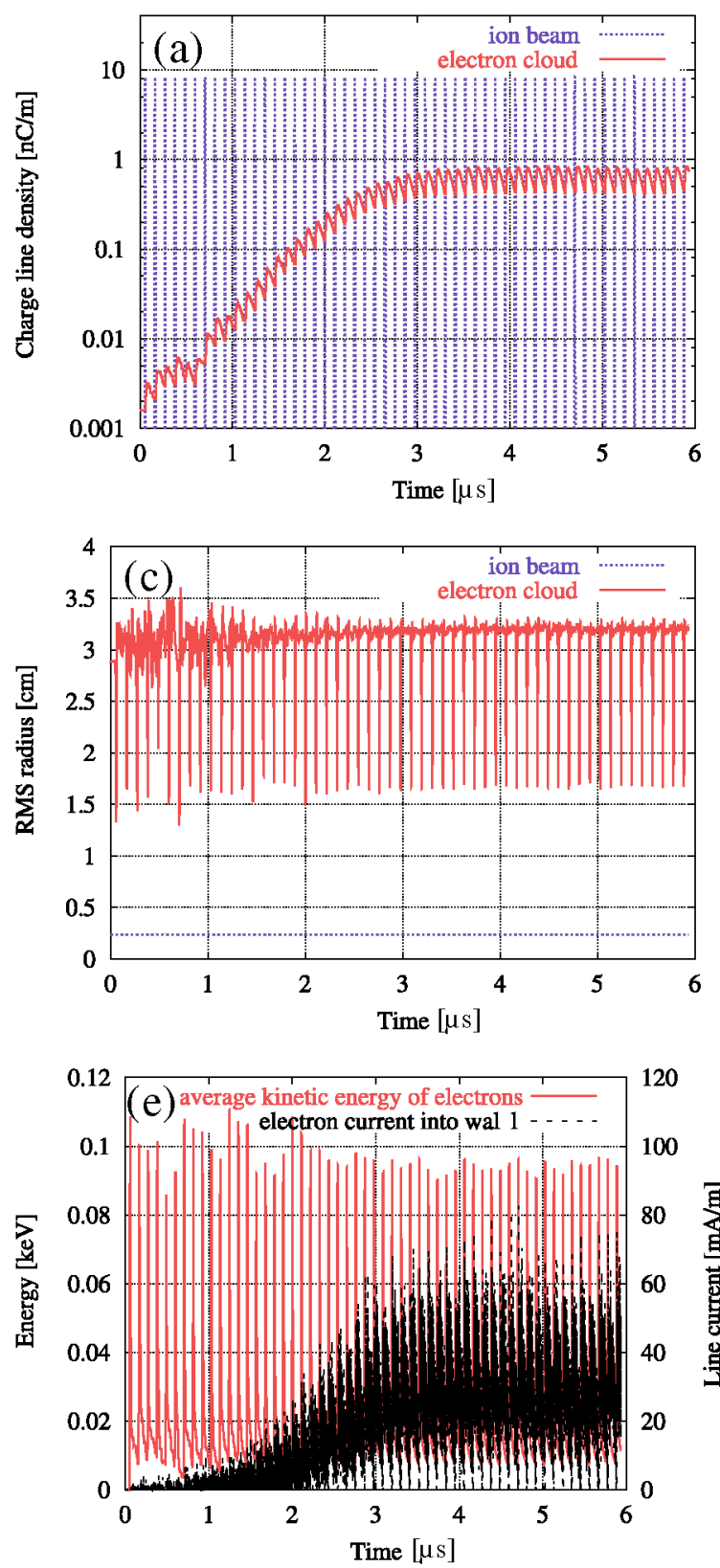

The distribution of the output angle $\theta_{\text {out }}$ is the same for reflected and secondary electrons and independent of the incident angle $\theta$, thus assuming a rough surface. The distribution of $\theta_{\text {out }}$ is given by

$$
P(\theta) d \theta \propto(\cos \theta)^{\alpha_{\theta}} \sin \theta d \theta,
$$

where the parameter $\alpha_{\theta}$ is an input parameter between zero (equivalent to black body radiation) and infinity $\left(\theta_{\text {out }}=\right.$ const $\left.=\pi / 2\right)$. The list of input parameters is shown in Table IV.
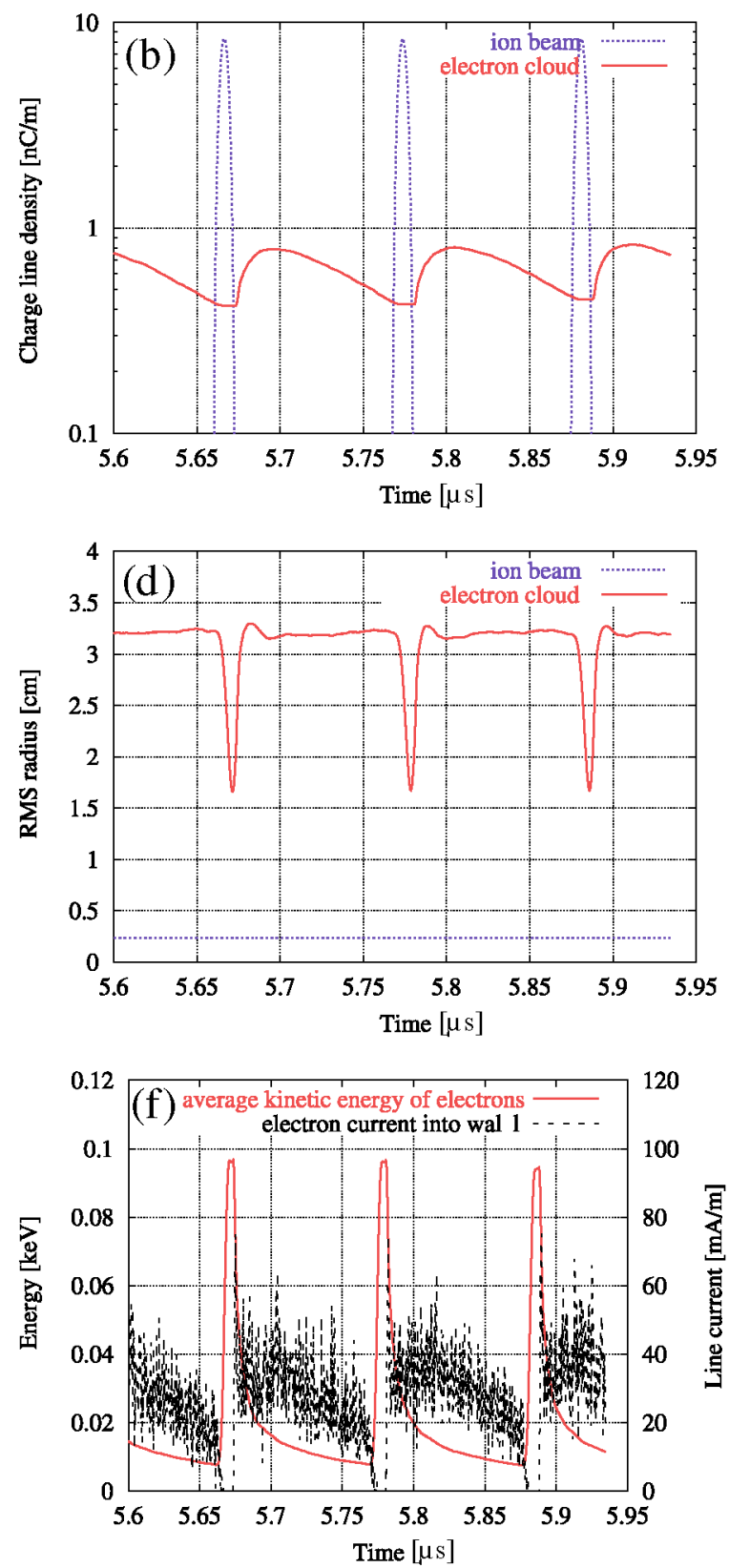

FIG. 5. (Color) Simulation results for the proton reference case. (a) Ion beam and electron cloud line density. (c) rms radius of ion beam and electron cloud. (e) Average kinetic energy of the electrons and current into the beam pipe wall. In parts (b),(d), and (f) the three last bunches from the 55 bunches in parts (a),(c), and (e) are shown, respectively. 
TABLE V. Maximum charge line density after 55 bunches and time after which saturation is reached in simulations under variation of input parameters. In each case only one parameter of the proton reference case is changed and the resulting line density is shown together with its relative change.

\begin{tabular}{|c|c|c|c|c|c|}
\hline Parameter & Unit & Value & $\begin{array}{c}\text { Change } \\
(\%)\end{array}$ & $\begin{array}{c}\lambda_{c e} \\
(\mathrm{nC} / \mathrm{m})\end{array}$ & $\begin{array}{c}\text { Saturation time } \\
(\mu \mathrm{s})\end{array}$ \\
\hline Reference case $p$ & $\cdots$ & $\cdots$ & $\cdots$ & 4.6 & 1.3 \\
\hline Bunch spacing & $\mathrm{ns}$ & 216 & +100 & 1.4 & 3.1 \\
\hline Beam offset & $\mathrm{mm}$ & 5 & $\cdots$ & 4.4 & 1.3 \\
\hline Beam radius & $\mathrm{mm}$ & 4.8 & +100 & 4.5 & 1.3 \\
\hline Pipe radius & $\mathrm{mm}$ & 60 & +50 & 4.5 & 1.7 \\
\hline$e$-gen./bunch & $\cdots$ & 50 & -50 & 4.6 & 1.4 \\
\hline$e$-gen./bunch & $\cdots$ & 1000 & +1000 & 4.6 & 1.4 \\
\hline$e$-gen./bunch & $\mathrm{mm}$ & 40 & +1660 & 4.6 & 1.4 \\
\hline Bunch length & $\mathrm{ns}$ & 18 & +28 & 4.6 & 1.4 \\
\hline Bunch length & $\mathrm{ns}$ & 10 & -28 & 4.6 & 1.3 \\
\hline Bunch shape $n$ & $\cdots$ & 1 & $\cdots$ & 4.1 & 1.3 \\
\hline Bunch shape $n$ & $\cdots$ & 6 & $\cdots$ & 4.6 & 1.1 \\
\hline Bunch charge & $\mathrm{nC}$ & 5 & -70 & 0.0 & $\cdots$ \\
\hline Bunch charge & $\mathrm{nC}$ & 7 & -50 & 1.3 & 3.3 \\
\hline Bunch charge & $\mathrm{nC}$ & 12 & -25 & 2.9 & 1.5 \\
\hline Bunch charge & $\mathrm{nC}$ & 20 & +25 & 5.6 & 1.1 \\
\hline Bunch charge & $\mathrm{nC}$ & 24 & +50 & 6.7 & 1.1 \\
\hline Bunch charge & $\mathrm{nC}$ & 32 & +100 & 7.3 & 1.1 \\
\hline$N_{\text {slices }}$ & $\cdots$ & 10000 & +100 & 4.5 & 1.2 \\
\hline$N_{\text {macro }}$, initial & $\cdots$ & 250 & +1000 & 4.6 & 1.3 \\
\hline Smoothing $d$ & $\mathrm{~mm}$ & 0.01 & -90 & 4.6 & 1.3 \\
\hline$\lambda_{c e}$, initial & $\mathrm{pC} / \mathrm{m}$ & 0.016 & -99 & 4.5 & 1.4 \\
\hline$P_{0}$ & $\ldots$ & 0.7 & -12 & 2.7 & 1.5 \\
\hline$P_{\infty}$ & $\cdots$ & 0.1 & -50 & 3.8 & 1.3 \\
\hline$E_{\text {reflect }}$ & $\mathrm{eV}$ & 80 & +33 & 5.7 & 1.1 \\
\hline$P_{\text {rediffuse }}$ & $\cdots$ & 0.4 & -20 & 5.6 & 1.1 \\
\hline$\delta_{\max }$ & $\cdots$ & 1.8 & -22 & 1.7 & 2.5 \\
\hline$\delta_{\max }$ & $\cdots$ & 2.0 & -13 & 2.7 & 1.6 \\
\hline$\delta_{\max }$ & $\cdots$ & 2.5 & +9 & 5.8 & 1.1 \\
\hline$E_{\max }$ & $\mathrm{eV}$ & 350 & +17 & 2.8 & 1.6 \\
\hline$E_{\text {secondary }}$ & $\mathrm{eV}$ & 30 & +50 & 9.2 & 1.1 \\
\hline$\alpha_{\delta}$ & $\cdots$ & 0.4 & -20 & 4.0 & 1.1 \\
\hline$\alpha_{\theta}$ & $\cdots$ & 0.0 & -100 & 4.0 & 1.2 \\
\hline$\alpha_{\theta}$ & $\cdots$ & 5.0 & +500 & 4.7 & 1.2 \\
\hline
\end{tabular}

\section{SIMULATION RESULTS}

Since the simulations have many input parameters and the result is very sensitive to changes in a number of those we first define reference cases for gold and proton beams. The reference cases should be close to worst case scenarios with respect to the beam parameters. We will then vary input parameters in one of the reference cases to find the sensitivity of the result with respect to these parameters.

The two reference cases are based on design intensities and short bunch spacing. The cases differ slightly in the charge per bunch and significantly in the bunch length. Furthermore, rest gas ionization is assumed for the proton case and loss-driven electron generation in the gold case. Beam and beam pipe sizes correspond to an assumed electron cloud in the whole machine for protons and in the warm regions only for gold. The two cases are listed in Table IV.

In Fig. 5 the simulation output is shown for the proton reference case. Figure 5(a) shows the ion beam and electron cloud charge line densities. After 25 bunches the electron cloud is saturated at approximately $0.3 \mathrm{nC} / \mathrm{m}$. The saturation is also visible in Fig. 5(b) which shows only the last three bunches. The saturation charge line density is comparable to expectations from the tune shift measurements (cf. Table III). However, the 
tune shift measurements were done at lower bunch charges.

Figures 5(c) and 5(d) show the transverse rms size of the ion beam and electron cloud for the whole bunch train and the last three bunches, respectively. The electron cloud size begins to drop when the bunch begins to pass through. During the bunch passage, electrons outside the bunch are accelerated towards the bunch center, and electrons in the bunch are trapped for the time of the passage. The electron cloud size begins to recover as soon as the bunch has passed. The electron cloud is much larger than the ion beam, and for the plateaus between bunch passages its rms size is consistent with an approximately uniform density. For a transverse uniformly distributed electron cloud, the rms size would be $r / \sqrt{2}=2.8 \mathrm{~mm}$, about $10 \%$ smaller than the value obtained in the simulation.

In Figs. 5(e) and 5(f) the average kinetic energy of the electrons and the electron current into the wall are shown. From this, an estimate of the heat load into the wall can be obtained. From Fig. 5(f) one finds an average kinetic energy of approximately $0.02 \mathrm{keV}$ and average electron current into the wall of about $30 \mathrm{~mA} / \mathrm{m}$. This corresponds to a heat load of the order of $0.1 \mathrm{~W} / \mathrm{m}$ or $300 \mathrm{~W}$ for the cold part of the ring, assuming that a large part of the not reflected electrons transforms their kinetic energy in heat. No increased heat load was observed during the tests in 2001. This may indicate that no electron clouds were formed in the cold regions. The minimum detectable heat load is $150 \mathrm{~W}$ [19].

The simulation of the gold beam reference case shows no significant increase in the initial electron line density. The final density after 55 bunches is 2 orders of magnitude smaller than in the proton reference case. This is largely due to the longer bunches and the reduced charge per bunch.

The sensitivity of the computed electron cloud density with respect to the input parameters was estimated by varying single input parameters only. This is shown in Table V. The simulation result is not sensitive to the number of longitudinal slices and the number of initial macroparticles as long as they are chosen large enough. The result is also insensitive to the number or location of electrons generated during a bunch passage. With an exponential electron multiplication process, the initial number of electrons is irrelevant if it is sufficiently large to start the process. The smoothing length $d$, which avoids singularities in the computation of the forces, can also be varied over a relatively large range without affecting the result.

However, the result is, to a varying degree, sensitive to almost all other parameters. Of the beam and machine parameters, the bunch spacing and the charge per bunch are the most important parameters, while changes in the beam offset, pipe radius, bunch length, and shape are of less consequence. Small variations in all of the surface parameters can change the result significantly. Thus a good knowledge of these values is required to achieve a good agreement between measurements and simulations. The simulation code was recently modified and results differ from those reported in Ref. [20].

\section{SUMMARY}

The signs of the measured coherent horizontal and vertical tune shifts along bunch trains in RHIC are consistent with the existence of electron clouds. From the measured tune shifts electron cloud densities were estimated. Electron cloud densities of the same order of magnitude could also be obtained in simulations with beam intensities somewhat higher than in the measurements. The cloud densities estimated from the tune shift measurements could not be reproduced with the bunch intensities in the measurement. Thus, physical effects may be missing in the simulation or there is an insufficient knowledge of the surface parameters. Simulations results were found to be sensitive to many input parameters.

\section{ACKNOWLEDGMENTS}

The authors would like to thank M. Bai, P. Cameron, M. A. Furman, M. Harrison, H.C. Hseuh, T. Kerner, W. MacKay, T. Nicoletti, F. Pilat, M. Pivi, T. Roser, A. Rossi, G. Rumolo, W.C. Turner, S. Tepikian, D. Trbojevic, S.Y. Zhang, and F. Zimmermann for support and discussions. This work was supported by the U.S. DOE under Contract No. DE-AC02-98CH10886.

[1] S. Y. Zhang, BNL Report No. C-A/AP/67, 2002.

[2] H.-C. Hseuh, L. Smart, and S. Y. Zhang, in Proceedings of the 2002 European Particle Accelerator Conference, Paris (EPS-IGA/CERN, Geneva, 2002).

[3] RHIC Design Manual, edited by H. Hahn, revision of October 2000, http://www.rhichome.bnl.gov/NT-share/ rhicdm/00_tocli.htm.

[4] G. Rumolo, F. Zimmermann, H. Fukuma, and K. Ohmi, in Proceedings of the 2001 Particle Accelerator Conference, Chicago (IEEE, Piscataway, NJ, 2001).

[5] G. Arduini, K. Cornelis, G. Ferioli, L. Jensen, and F. Zimmermann, in Proceedings of the 2000 European Particle Accelerator Conference, Vienna (EPS-IGA/ CERN, Geneva, 2000).

[6] G. Arduini, K. Cornelis, W. Höfle, G. Rumolo, and F. Zimmermann, in Proceedings of the 2001 Particle Accelerator Conference, Chicago (Ref. [4]).

[7] W. Fischer, M. Bai, J. M. Brennan, M. Blaskiewicz, P. Cameron, H.C. Hseuh, H. Huang, W. MacKay, T. Roser, T. Satogata, L. A. Smart, D. Trbojevic, and S.Y. Zhang, in Proceedings of the 2002 European Particle Accelerator Conference, Paris (Ref. [2]). 
[8] P. Cameron, R. Connolly, A. Drees, T. Ryan, H. Schmickler, T. Shea, and D. Trbojevic, BNL Report No. RHIC/AP/155, 1998.

[9] M. A. Furman and A. A. Zholents, in Proceedings of the 1999 Particle Accelerator Conference, New York (IEEE, Piscataway, NJ, 1999).

[10] F. Zimmermann, in Proceedings of the 2001 Particle Accelerator Conference, Chicago (Ref. [4]).

[11] K. Ohmi, S. Heifets, and F. Zimmermann, in Proceedings of the 2001 Asian Particle Accelerator Conference, Beijing [CERN Report No. SL-2001-062 (AP), 2001].

[12] M. A. Furman (private communication).

[13] M. Blaskiewicz, computer program NCSEC (for Nearly Cylindrically Symmetric Electron Cloud), BNL, 2000.

[14] M. Blaskiewicz, M. A. Furman, M. Pivi, and R. J. Macek, Phys. Rev. ST Accel. Beams (to be published).
[15] V. Danilov, A. Aleksandrov, M. Blaskiewicz, and J. Wei, in Proceedings of the 2001 Particle Accelerator Conference, Chicago (Ref. [4]).

[16] M. A. Furman and M. Pivi, LBNL Report No. LBNL49711, CBP Note-415, 2002.

[17] B. Henrist, N. Hilleret, M. Jimenez, C. Scheuerlein, M. Taborelli, and G. Vorlaufer, in Proccedings of the ECLOUD'02 Workshop at CERN (CERN Yellow Report No. CERN-2002-001, 2002).

[18] V. Baglin, I. Collins, B. Henrist, N. Hilleret, and G. Vorlaufer, CERN LHC Project Report No. 472, 2002.

[19] T. Nicoletti (private communication).

[20] W. Fischer, J. M. Brennan, M. Blaskiewicz, and T. Satogata, in Proccedings of the ECLOUD'02 Workshop at CERN (Ref. [17]), BNL Report No. C-A/ AP/71, 2002. 